EPJ Web of Conferences 19, 06001 (2012)

DOI: $10.1051 /$ epjconf/20121906001

(C) Owned by the authors, published by EDP Sciences, 2012

\title{
Inner galactic disc metallicity distributions and the bulge-disc relation
}

\author{
V. Hill1,a , C. Babusiaux², A. Gómez² ${ }^{2}$ M. Haywood ${ }^{2}$, D. Katz ${ }^{2}$ and F. Royer ${ }^{2}$ \\ 1 Université de Nice Sophia-Antipolis, CNRS, Observatoire de la Côte d'Azur, BP. 4229, \\ 06304 Nice Cedex 4, France \\ 2 GEPI, Observatoire de Paris, Pl. J. Janssen, 92195 Meudon Cedex, France
}

\begin{abstract}
The intermediate-to-old population of the inner galactic disk remains a relatively uncharted territory. We present the results of a high resolution spectroscopic survey of over 200 red clump stars in the inner galactic disc ( $3-5 \mathrm{kpc}$ from the galactic centre, $\mathrm{Z} \leq 300 \mathrm{pc}$ from the plane). The metallicity distribution of the sample is clearly more metal-rich than the solar neighborhood, implying a significant radial gradient in the galactic disc at the intermediate ages probed by red clump stars. We discuss the implications of these results on the existence of gradients and their flattening with age. Finally, for the first time, this result brings support to the idea that the metal rich part of the bulge MDF may originate from the inner disk through dynamical instabilities.
\end{abstract}

\section{SAMPLE CONTENT}

The sample analysed here consists of stars selected to be red clump stars along two line of sights close to the galactic center, at galactic coordinates $(1=0.1, \mathrm{~b}=-2.0 \mathrm{deg})$ and $(1=-2.7, \mathrm{~b}=-3.6 \mathrm{deg})$. The selection was performed in the reddening free reduced $\mathrm{K}_{\mathrm{s}}^{\prime}$ magnitude (see e.g. Babusiaux \& Gilmore 2005 [1]), versus combinations of V,I,J,H,K colours, and is illustrated in Fig. 1 for the $(1=0.1$, $\mathrm{b}=-2.0 \mathrm{deg}$ ) field, where the disc red clump stars sequence clearly stand out as a brighter (and bluer) extension of the bulge red clump. In this plane, assuming an intrinsic magnitude and colour for the red clump, the $\mathrm{K}_{\mathrm{s}}^{\prime}$ of these stars can be read off as distance, and the colour is related to the extinction (assuming also a extinction law). Distances and $A_{V}$ are also indicated on the figure to illustrate the expected extent of the sample in distance: from 1 to $5 \mathrm{kpc}$ from the sun.

We obtained high-resolution spectra with FLAMES/GIRAFFE at the VLT for $\sim 120$ stars located in each of the two fields, and derived radial velocities as well as fully spectroscopic stellar parameters (effective temperature, gravity, [Fe/H]), using the same method as in Hill et al. (2011, [2]). Distances to the targets where then determined by comparison to Padova isochrones. After trimming out stars with radial velocity variations, low $\mathrm{S} / \mathrm{N}$ and other problematic spectra, we were left with a total sample of 207 stars with radial velocities, of which 172 have good quality parameters.

The samples in the two fields are expected to be dominated by thin disc stars, but owing to their different galactic latitudes, we expect the field furthest from the plane to contain the largest contribution from the thick disc. We could confirm that the $(1=0.1, b=-2 \mathrm{deg})$ field, which reached only to $\mathrm{Z} \sim 140 \mathrm{pc}$ below the plane at a distance of $4 \mathrm{kpc}$, does not contains any significant thick disc population, while the field $(1=-2.7, b=-3.6 \mathrm{deg})$ contains metal-poor stars with a higher velocity dispersion. Indeed, a gaussian decomposition of the metallicity-velocity distribution reveals two populations in this

\footnotetext{
a e-mail: Vanessa.Hill@oca.eu
}

This is an Open Access article distributed under the terms of the Creative Commons Attribution-Noncommercial License 3.0, which permits unrestricted use, distribution, and reproduction in any noncommercial medium, provided the original work is properly cited. 


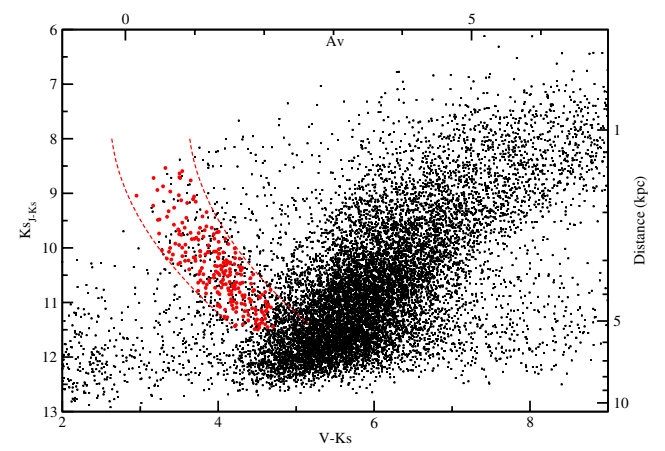

Figure 1. Reduced $\mathrm{K}_{\mathrm{s}}^{\prime}$ magnitudes vs. (V-K) colours of the field at $(1=0.1, \mathrm{~b}=-2.0 \mathrm{deg})$, with our sample selection over-plotted as red circles.

latter field, a majority of metal-rich stars $(<[\mathrm{Fe} / \mathrm{H}]>=+0.14))$ with low radial velocity dispersion $\left(\sigma_{\mathrm{Vrad}}=52 \pm 4 \mathrm{~km} / \mathrm{s}\right)$, and a minority metal-poor component $\left.(<[\mathrm{Fe} / \mathrm{H}]\rangle=-0.61\right)$ with high velocity dispersion $\left(\sigma_{\mathrm{Vrad}}=88 \pm 18 \mathrm{~km} / \mathrm{s}\right)$, accounting for $\sim 11 \pm 4 \%$ of the sample. In addition, this high radial velocity dispersion population is predominantly found at large distances from the sun ( $\geq 3.5 \mathrm{kpc}$ ) where this line of sight probes out to $Z \geq 220 \mathrm{pc}$ from the plane (see also Fig. 3).

When both line of sights are combined, a total of $\sim 8 \pm 2 \%$ of the total sample resides in this metalpoor warm component that we tentatively associate with the thick disc, to be confirmed by e.g. $[\alpha / \mathrm{Fe}]$ measurements in the sample. In any case, the present sample therefore consists of $92 \pm 2 \%$ of thin disc stars, and is therefore well suited to study metallicity distributions and gradients in the inner thin disc.

\section{THE METALLICITY DISTRIBUTION}

The metallicity ([Fe/H]) distribution (MDF) for the sample is displayed in Fig. 2. In both fields, a very prominent metal-rich peak is found, around $[\mathrm{Fe} / \mathrm{H}]=+0.15$. To avoid systematics in the comparison of this distribution to the solar neighborhood MDF, we re-analysed a subsample of 50 stars from the Elodie spectral archive ${ }^{1}$ (Moultaka et al. (2004, [3])), selected from the study of 177 red clump giants in the solar neighbourhood by Mishenina et al. (2006, [4]). We analysed these stars using the same wavelength coverage, resolution and method as for our inner disc sample, and found that our metallicities are on the same scale as Mishenina et al. (2006, [4]) in the metal-rich regime, while in the metal-poor regime, we tend to overestimate our metallicities by $\sim 0.2 \mathrm{dex}$. We corrected for this biais in the comparison to the solar neighborhood MDF shown in Fig. 2: the inner disc appears clearly more metal-rich than the solar neighborhood by $\sim 0.2$ dex.

We further compare in the lower panel of Fig. 2 the MDF of the inner disc to the MDF of red clump stars in the bulge in Baade's Window $(1=0, b=-4 \mathrm{deg})$ (Hill et al. 2011 [2]). The galactic bulge has been suggested to host two kinematically and chemically distinct populations (Babusiaux et al. 2010 [5] and Hill et al. 2011 [2]) of roughly equal weight at the latitude of Baade's window: a metal-rich component $(<[\mathrm{Fe} / \mathrm{H}]>\sim+0.3))$ with a high radial velocity dispersion and a vertex deviation as well as low $[\mathrm{Mg} / \mathrm{Fe}]$ ratios, and a metal poor population $(<[\mathrm{Fe} / \mathrm{H}]>\sim-0.3)$ showing a somewhat lower velocity dispersion, no vertex deviation and high $[\mathrm{Mg} / \mathrm{Fe}]$ ratios. The metal-rich population is associated to the galactic bar, while the metal-poor population would be a spheroidal component of the bulge. In this scheme, the observed metallicity gradient observed along the minor axis for $b \geq 3-4$ deg (Zoccali et al. 2008 [6], Gonzales et al. 2011 [7]) is associated to the gradual disappearance of the bar population

\footnotetext{
${ }^{1}$ http://atlas.obs-hp.fr/elodie/
} 


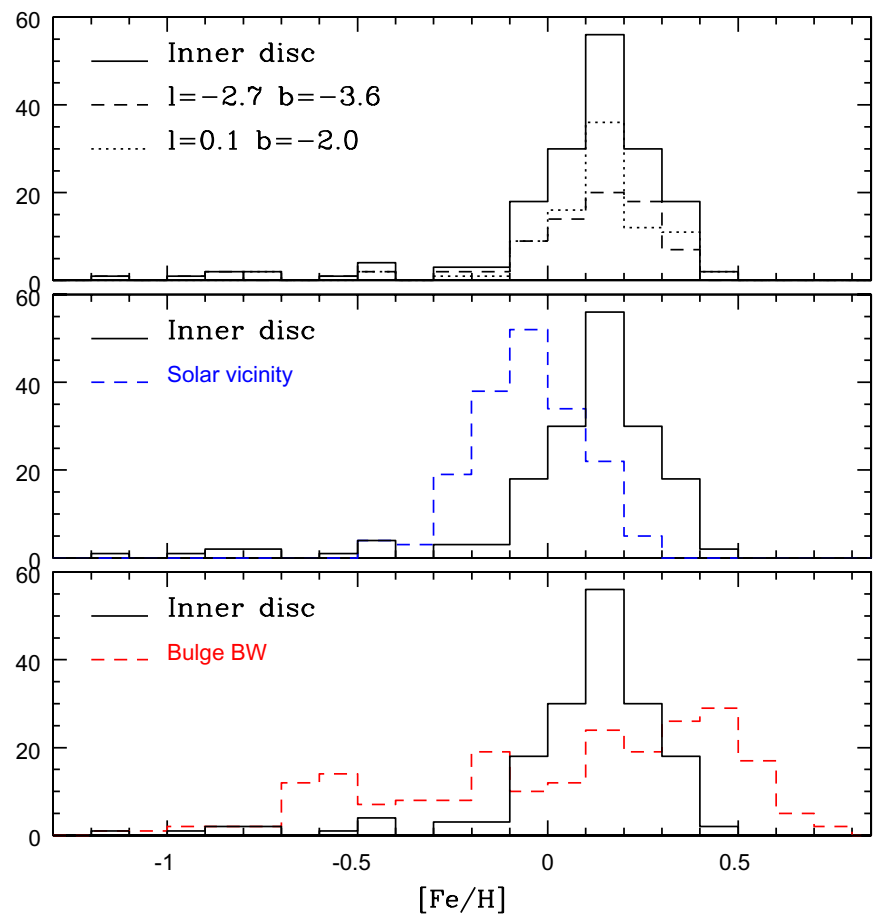

Figure 2. Metallicity distribution of the inner disc samples (solid black line). In the upper panel, the two individual fields at galactic coordinates $(1=-2.7, b=-3.6$, dashed line $)$ and $(1=0.1, b=-2.0$, dotted line $)$ are compared. The total distribution (solid black line) is shown with the MDF in the solar neighborhood (as of Mishenina et al. 2006 [4], corrected to march our metallicity scale, dashed blue line) in the middle panel, and to the galactic bulge in Baade's Window (Hill et al. 2011 [2], dashed red line) in the lower panel.

at large distances from the plane. The inner disc sample presented here shows that the metal-rich bulge component bears a direct relation to the inner regions of the galactic disc, with a mean metallicity directly continuing the metallicity gradient between the solar neighborhood and inner disc, as expected if this component is a bar formed from disc material through dynamical instabilities.

\section{GRADIENT TOWARDS THE INNER GALACTIC DISC}

Armed with the distances determined from the spectroscopic parameters for our sample stars, we can attempt to measure the radial gradient towards the inner parts of the galactic disc. In Fig. 3, we show the metallicity as a function of the distance to the targets of our sample, as well as median metallicities in four distance bins. One has to bear in mind that the furthest distance bin (D > $4.3 \mathrm{kpc}$ ) and probably also the second to last (3.3 to $4.3 \mathrm{kpc}$ ) include a significant proportion of thick disc stars (see Sec. 1), and that beyond 3.5 to $4 \mathrm{kpc}$, our selection criterion to avoid background bulge RGB stars probably biasses our sample against the most metal-rich (reddest) disc red clump stars. Given these two sources of biasses, the metallicity distribution beyond $3.5 \mathrm{kpc}$ has to be viewed with extreme caution.

In the nearer distance range where we expect no bias to occur ( 1.5 to $3.5 \mathrm{kpc}$ ), there is no sign of evolution of the metallicity with distance, and the sample has a median $<[\mathrm{Fe} / \mathrm{H}]>=+0.17$. Comparing this median (at a median distance of $2.5 \mathrm{kpc}$ ) to the solar neighborhood median metallicity for red clump stars (Mishenina et al. 2006 [4], corrected to our metallicity scale), the gradient that can be inferred is of $\delta[\mathrm{Fe} / \mathrm{H}] / \delta \mathrm{D}=+0.092 \mathrm{dex} / \mathrm{kpc}$. However, since there is no sign of metallicity change within the range $\mathrm{D}=1.5$ to $3 \mathrm{dex} / \mathrm{kpc}$, it is tempting to argue rather for a step-function (of 


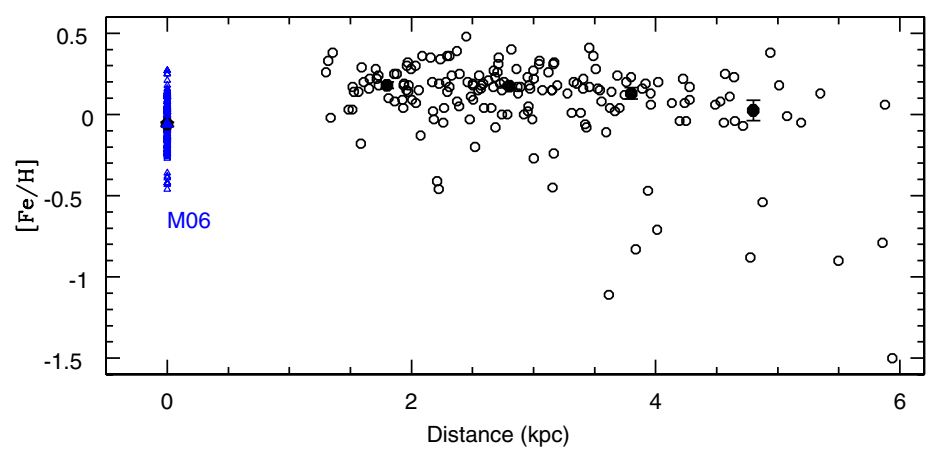

Figure 3. Metallicity versus distance of our targets (open black symbols). The local sample of Mishenina etal. (2006 [4]), corrected to our metallicity scale, is also plotted. The large filled symbols with errors show the mean metallicities in five distance bins.

$\delta[\mathrm{Fe} / \mathrm{H}]=+0.23 \mathrm{dex}$ ) somewhere around $\mathrm{D}=1-1.5 \mathrm{kpc}$ (or galactocentric radii of $\mathrm{R}_{\mathrm{GC}} \sim 6.5-7 \mathrm{kpc}$ ), rather than a smooth gradient between the solar neighborhood and the inner parts of the galactic disc. It is not the first time that such a behaviour is advocated for the inner disc. Evidence for a steeper gradient towards the inner galactic disc than towards the outer parts is supported by observations of cepheids (e.g. Pedicelli et al. 2009 [8] finds a slope of slope $\delta[\mathrm{Fe} / \mathrm{H}] / \delta R_{\mathrm{GC}}=-0.130 \pm 0.015 \mathrm{dex} / \mathrm{kpc}$ in the inner galaxy, whereas the gradient if of $-0.042 \pm 0.005 \mathrm{dex} / \mathrm{kpc}$ outside the solar circle). Observations of open clusters in the inner galaxy by Magrini et al. (2010 [9]) also argue for $\delta[\mathrm{Fe} / \mathrm{H}] / \delta R_{\mathrm{GC}}=$ $-0.137 \pm 0.041$ dex. Both datasets would however be just as consistent with a step function around $\mathrm{R}_{\mathrm{GC}} \sim 6.5 \mathrm{kpc}$.

Finally, evidence for gradient flattening with age (or steepening with time) has been advocated by Stanghellini \& Haywood (2010 [10]), from Planetary Nebulae (PNe) classified in several age groupings. Because our sample is selected as red clump giant stars, it is expected to contain a mix of stellar ages that can a priori span between $\sim 0.5$ and 13 Gyrs. Girardi \& Salaris 2001 [11] however argue that, owing to the masses and lifetimes for stars that pass through the He-burning phase, for a constant star formation rate, the age distribution of red clump stars in fact reflect rather the star formation of the last 1-2 Gyrs. Our sample should therefore sample a mix of ages that may vary as a function of $\mathrm{R}_{\mathrm{GC}}$ according to the star formation rate, but our sample should however be significantly older than Cepheids, or the youngest open clusters considered in Magrini et al. (2010 [9], < 0.8 Gyr). Our shallower gradient could then tentatively be taken as a flattening of the gradient with age, although it is clear that the mere shape of the gradient (linear or step-function) has to be further constrained before concluding firmly.

\section{References}

[1] C. Babusiaux, G. Gilmore, MNRAS 358, 1309 (2005)

[2] V. Hill, A. Lecureur, A. Gómez, M. Zoccali, M. Schultheis, C. Babusiaux, F. Royer, B. Barbuy, F. Arenou, D. Minniti et al., A\&A 534, A80 (2011)

[3] J. Moultaka, S.A. Ilovaisky, P. Prugniel, C. Soubiran, PASP 116, 693 (2004)

[4] T.V. Mishenina, O. Bienaymé, T.I. Gorbaneva, C. Charbonnel, C. Soubiran, S.A. Korotin, V.V. Kovtyukh, A\&A 456, 1109 (2006)

[5] C. Babusiaux, A. Gomez, V. Hill, F. Royer, M. Zoccali, F. Arenou, A\&A 519, A77 (2010)

[6] M. Zoccali, V. Hill, A. Lecureur, B. Barbuy, A. Renzini, D. Minniti, A. Gómez, S. Ortolani, A\&A 486, 177 (2008)

[7] O.A. Gonzalez, M. Rejkuba, M. Zoccali, E. Valenti, D. Minniti, A\&A 534, A3 (2011) 
Assembling the Puzzle of the Milky Way

[8] S. Pedicelli, G. Bono, B. Lemasle, P. François, M. Groenewegen, J. Lub, J.W. Pel, D. Laney, A. Piersimoni, M. Romaniello et al., A\&A 504, 81 (2009)

[9] L. Magrini, S. Randich, M. Zoccali, L. Jilkova, G. Carraro, D. Galli, E. Maiorca, M. Busso, A\&A 523, A11 (2010)

[10] L. Stanghellini, M. Haywood, ApJ 714, 1096 (2010), 1003.0759

[11] L. Girardi, M. Salaris, MNRAS 323, 109 (2001) 